\title{
Temporospatial Relationship of Lipid Droplets and Mitochondria in Cardiac Muscle by Focused Ion Beam Scanning Electron Microscopy.
}

\author{
Tsengming Chou ${ }^{1}$, Carole Sztalryd-Woodle ${ }^{2}$, and Ru-ching Hsia ${ }^{3}$ \\ 1. Laboratory for Multiscale Imaging, Stevens Institute of Technology, Hoboken, USA \\ 2. Department of Medicine, University of Maryland Baltimore, Baltimore, USA \\ 3. Electron Microscopy Core Imaging Facility, University of Maryland Baltimore, Baltimore, USA
}

Obesity is a rising health problem in the United States and many developed countries. The World Health Organization estimates that, in 2014, more than 1.9 billion adults were overweight worldwide, and more than 600 millions were obese [1]. Obesity is the result of an imbalance in lipid homeostasis and is evidenced by the excessive accumulation of cytoplasmic lipid droplets (CLDs) in cardiac muscles. The CLD is a dynamic organelle that interacts with various cell organelles such as the endoplasmic reticulum, endosomes and mitochondria. Our previous studies suggested that the LD specific protein, perilipin 5, is involved in promoting association of mitochondria to LDs [2]. Here we investigate the temporospatial relationship of LDs, mitochondria, endoplasmic reticulum (sarcoplasmic reticulum) and muscle fibrils in a 3D volume of mouse cardiac muscle using a focused ion beam scanning electron microscope (FB-SEM).

Transgenic mice over-expressing perilipin 5 under the $\alpha$-myosin heavy chain promoter were fasted for $24 \mathrm{hr}$ to increase the number of LDs before euthanasia. Heart muscles were excised and manually trimmed into thin slices, fixed, post stained with osmium tetroxide, dehydrated and embedded in spurs epoxy resin. Epoxy resin blocks containing heart muscle were trimmed and sectioned using an ultramicrotome to expose tissue surface. The block faces were glued onto a SEM-stub, painted with conductive paint, sputter-coated and loaded onto a Zeiss Auriga FIB/SEM. Back scattered SEM images were collected at a beam energy of $1.5 \mathrm{kV}$, and ion beam millings were done at $1 \mathrm{nA}$ and $20 \mathrm{~nm}$ per slice. A total muscle tissue volume of $\sim 15 \times 24 \times 12 \mu \mathrm{m}^{3}$ was analyzed in this study. Image alignment, segmentation and 3D analyses were done by using ImageJ and Avizo 9.0 software.

LDs were present in abundance in cardiac muscles of transgenic mice. LDs were closely associated with mitochondria and the presence of LDs disrupted the normal distribution of mitochondria along the muscle fibrils. Vesicles of unknown function were observed in mitochondria in the heart muscle of transgenic animals, but not in wild type mice. Although it is unclear whether this is related to altered mitochondrial function in these mice, it is noteworthy that these vesicles appeared to originate between mitochondrial inner membrane stacks. A complete segmentation of each key organelle in the entire volume will be necessary to reveal the spatial relationship and interactions among the key organelles. Moreover, the membrane interactions predicted between LDs and between mitochondria, LDs and the sarcoplasmic reticulum may require higher magnification imaging.

\section{References:}

[1] World Health Organization Fact sheet $\mathrm{N}^{0} 311$. http://www.who.int/mediacentre/factsheets/fs311/en/

[2] H Wang et al, Journal of Lipid Research 52 (11), p. 2159.

[3] This research effort used microscope resources partially funded by the National Science Foundation through NSF Grant DMR-0922522. 

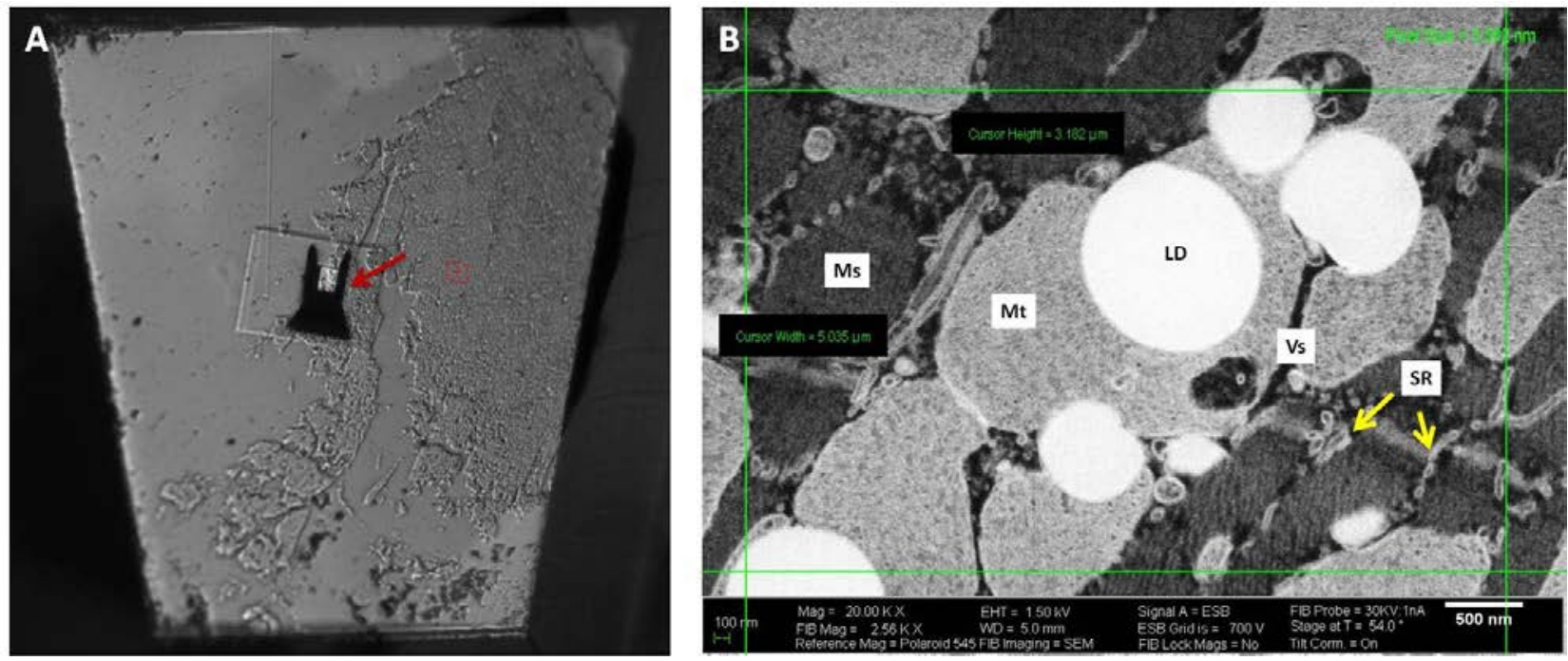

Figure 1. The epoxy resin block face showing the exposed cardiac muscle tissue (A) and the back scattered SEM image (B) (LD, lipid droplet; Mt, mitochondrion; Ms, muscle fibril; SR, sarcoplasmic reticulum; Vs, vesicle of unknown function). Red arrow points to the excavated trench and the milled area. Yellow arrows point to the SR network.
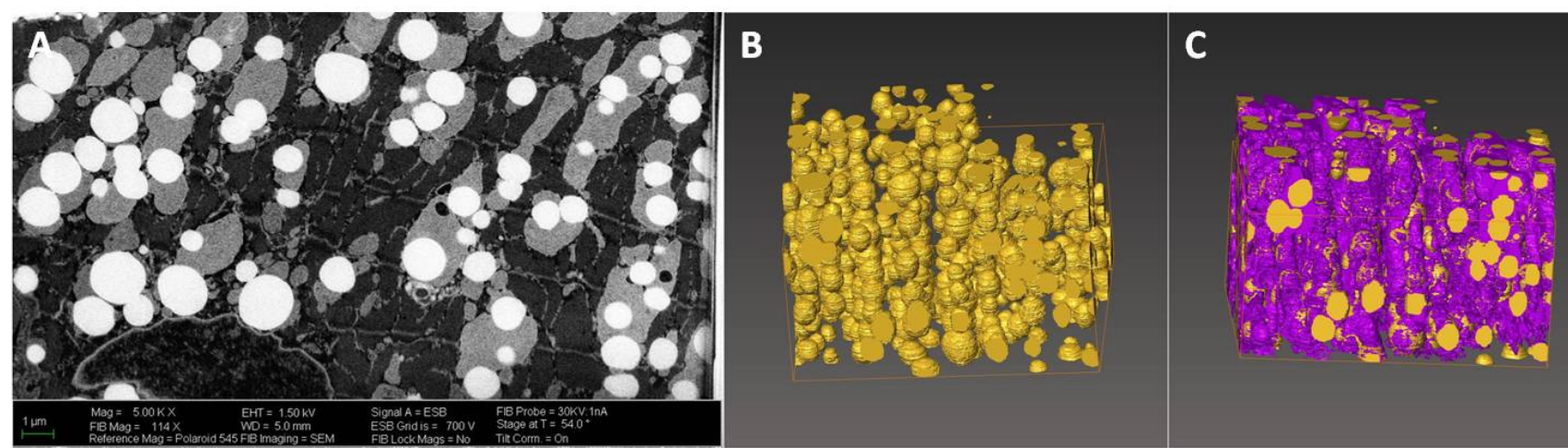

Figure 2. Single SEM slice demonstrating the close association of LDs with mitochondria (A). LDs and mitochondria were segmented using thresholding (B\&C). B shows the 3D distribution of LDs (gold color); C shows a combined image of LDs and mitochondria (purple) in the 3D volume. 